\title{
THE PARCELLATION OF THE MEDIAL GENICULATE BODY OF THE CAT DEFINED BY THE AUDITORY RESPONSE PROPERTIES OF SINGLE UNITS ${ }^{1}$
}

\author{
MICHAEL B. CALFORD
}

Department of Physiology and Pharmacology, University of Queensland, St. Lucia, Queensland, Australia

Received March 21, 1983; Revised June 13, 1983; Accepted June 13, 1983

\begin{abstract}
Single unit responses were recorded in the medial geniculate body (MGB) of anesthetized cats. In response to acoustical stimulation the properties of response latency, discharge pattern, frequency tuning, binaural interaction, and habituation were examined to allow an appraisal of the differentiation of the MGB by electrophysiological means. It is found that definite boundaries can be determined at which there is a distinct change in response properties; the position of these "physiological boundaries" seems to correspond with the boundaries between the seven subnuclei of the MGR described by Morest (Morest, D. K. (1964) J. Anat. 98: 611-630) in Golgi-stained material. Using these physiological boundaries to determine unit locations, population comparisons are made allowing the description of each subnucleus in terms of its auditory response properties. It is suggested that these properties, together with the limited information gained from Nissl cytoarchitecture, are sufficient to describe the location of physiological recording sites in the MGB.
\end{abstract}

The medial geniculate body $(\mathrm{MGB})^{2}$ is a thalamic relay of the mammalian auditory pathway. It receives bilateral projections from the inferior colliculus (IC; Andersen et al., 1980b; Kudo and Niimi, 1978, 1980; Moore and Goldberg, 1963; Oliver and Hall, 1978a, b; Powell and Hatton, 1969) and projects to, and receives projections from, the auditory cortical fields (Andersen et al., 1980a, c; Diamond et al., 1958, 1968, Merzenich and Colwell, 1975; Niimi and Matsuoka, 1979; Rose and Woolsey,

${ }^{1}$ I am grateful for the help of Lindsay Aitkin in all aspects of this work. The generosity of Bill Webster in providing help with some of the early experiments and in the use of his laboratory is gratefully acknowledged. I also thank Dexter Irvine and other members of the Department of Psychology at Monash University, Clayton, Victoria, Australia, for the use of laboratory facilities. This work has benefitted from advice and help given by my colleagues: Mal Semple, Lisa Wise, Jack Pettigrew, Dennis Phillips, and Chris Kenyon. For their technical help and expertise I thank Jill Poynton, Karen Styles, Judy Sack, and Lynne Hepburn.

This study has been supported by funds from the Australian Research Grants Scheme.

${ }^{2}$ The abbreviations used are: BIC, brachium of the inferior colliculus; BIN, nucleus of the BIC; DC, caudodorsal nucleus of the medial geniculate body; Dd, deep dorsal nucleus of the medial geniculate body; $\mathrm{E}$, excitatory; F, facilitatory; I, inhibitory; IC, inferior colliculus; LV, pars lateralis of the medial geniculate body; MGB, medial geniculate body; MGM, medial division of MGB; MGm, magnocellular division of $\mathrm{MGB}$; NS, suprageniculate nucleus of MGB; $\mathrm{O}$, no response; $\mathrm{OV}$, pars ovoidea of MGB; VL, ventrolateral nucleus of MGB.
1949, 1958; Winer et al., 1977). The MGB of the cat has been divided into at least seven subnuclei on the basis of cellular morphology in Golgi-stained material (Morest, 1964, 1965a; Ramòn y Cajal, 1955); using current terminology these are: pars lateralis (LV) and pars ovoidea (OV) of the ventral division (MGV), caudodorsal (DC), deep dorsal (Dd), and suprageniculate (NS) nuclei of the dorsal division, the ventrolateral nucleus (VL), and the medial division (MGM) (Andersen et al., 1980a, b; Calford and Webster, 1981).

Most electrophysiological studies have been concerned only with the properties of units in the ventral division. Units in LV and OV have short latencies to acoustic stimuli and are sharply tuned to tonal frequency; LV has been shown to have a laterocaudoventral to mediorostrodorsal/low- to high-unit best frequency (BF) tonotopic organization (Aitkin and Webster, 1972; Calford and Webster, 1981). Units in MGM are reported to show a wide range of tuning properties, from extremely broad to as sharp as MGV (Aitkin, 1973; Phillips and Irvine, 1979), and most units in DC show extremely broadtuning characteristics and respond at long latencies (Calford and Webster, 1981).

The present study compares the auditory response properties of units in different subdivisions of the MGB of the anesthetized cat. It is found that cytoarchitectonic borders between subnuclei can be correlated with changes in physiological properties and that differences 
in unit responses can be demonstrated between subnuclei on a population basis. These findings will form the physiological basis on which the differential ascending input to the MGB from the inferior colliculus will be examined in the subsequent paper (Calford and Aitkin, 1983). Preliminary reports of some of this material have been published elsewhere (Aitkin et al., 1981; Calford et al., 1980).

\section{Materials and Methods}

Electrophysiological data collected from 31 cats are presented in the present paper, while anatomical material derived from the retrograde transport of horseradish peroxidase (HRP) from iontophoretic injections into the MGB will be reported in the following paper (Calford and Aitkin, 1983). Only clearly isolated single units were used in the electrophysiological analysis.

All cats were free of middle ear pathology as far as could be determined by otoscopic examination. The first 11 cats were anesthetized initially with sodium pentobarbital (Nembutal, $40 \mathrm{mg} / \mathrm{kg}$, i.p.) and maintained with small doses of ketamine hydrochloride (Ketalar, $10 \mathrm{mg}$, i.m.). The remaining 20 cats were anesthetized by injection of 3 parts Ketalar to 1 part Rompun (xylazine hydrochloride), at a dose rate of $0.5 \mathrm{ml} / \mathrm{kg}$. Supplementary doses of half the initial strength were administered at 1 to 2 hourly intervals. After surgery and during recording Ketalar was the dominant anesthetic in both cases.

Cats were positioned in a Trent Wells stereotaxic frame with blunt ear bars. Surgery for the first 11 cats was as previously described (Calford and Webster, 1981). Briefly, the trachea was cannulated, the pinnae were removed, and a sealed sound delivery system, incorporating a probe tube, was inserted into each meatus.

With the remaining 20 cats the trachea and pinnae were left intact and sound was delivered through calibrated hollow ear bars; these animals were also used in HRP tracing experiments. For both groups of animals an area of bone about $5 \mathrm{~mm}$ in diameter, immediately anterior to the tentorium and lateral to the midline, was removed and the dura was reflected. Restricted aspiration of cerebral and hippocampal structures exposed the dorsal, lateral, and caudal surfaces of the MGB.

Micropipettes, blunted to a 3- to 5- $\mu \mathrm{m}$ tip diameter and filled with either $2 \mathrm{~mol} /$ liter of $\mathrm{NaCl}$ (saturated with pontamine sky blue dye) or 10\% HRP (Sigma Chemical Co., type VI) in physiological saline, were held in a microdrive and advanced in frontal or parasagittal planes onto the MGB surface.

Stimuli were generated as previously described (Calford and Webster, 1981). The system provided independent control of two channels, with standard stimuli consisting of tone or white noise bursts of $300 \mathrm{msec}$ duration and $4 \mathrm{msec}$ rise and fall periods presented at a rate of 1 $\mathrm{Hz}$. During a recording session these stimuli were varied frequently; variation included changes in burst length and repetition rate, varying phase between channels, and the presentation of click stimuli.

Extracellular action potentials were detected via a high input-impedance preamplifier and led to an amplifier, giving a total gain of 1000 . The amplified signal was viewed on a dual-trace Tektronix storage oscilloscope, as well as being acoustically monitored. An adjustable Schmitt trigger could be set to give a pulse in response to a selected action potential. This allowed the computation and presentation of response histograms "on-line" by a Nova 2 computer interfaced to a VT55 video terminal and a Data General digital magnetic tape recorder.

Histological procedures. Brains were fixed by transcardiac perfusion with either $10 \%$ formal/saline or $2.5 \%$ glutaraldehyde, and $50-\mu \mathrm{m}$ parasagittal or near-frontal sections were cut on a Leitz freezing microtome. Mounted sections were stained with thionine or, if processed for the HRP reaction product, were counterstained with neutral red. Sections were viewed on a Leitz micropromar or on a profile projector (Zeiss or Nikon) and electrode tracks were identified by the location of blue spots produced by the extrusion of pontamine sky blue (electrode negative; $25 \mu \mathrm{A}$ for $10 \mathrm{~min}$ ) or by the location of HRP reaction product. The positions of all units were marked upon reconstructed electrode tracks by reference to the depth at which they were encountered. Relevant thionine-stained sections were traced and the positions of boundaries of MGB subnuclei were indicated, using the criteria outlined below.

Recognition of divisions of Nissl-stained sections. Although the exact position of the boundaries between divisions is difficult to specify in Nissl-stained sections, it is generally possible to identify the major subnuclei of the MGB. These areas are differentiated by variations in the sizes of Nissl-stained cells and their packing densities.

There is some difficulty in identifying cell groups in isolated sections until the three-dimensional organization of the subdivisions can be recognized. For this purpose a series of four near-frontal sections, showing the positions of the major subnuclei of the MGB, is presented in Figure 1. It is obvious that distinctions between nuclei are not always prominent and boundaries are not always clear.

In the most caudal sections at which the MGB appears (A3.5 in Fig. 1), the entire protrusion is occupied by the caudal extent of DC, which is separated from the nucleus of the brachium of the IC (BIN) by the fibers of the accessory optic tract. At the level of the posterior third of the MGB (A5.0 in Fig. 1), the complex has expanded and all of the major subdivisions are found. The caudodorsal nucleus (DC) can be distinguished from the ventral division (MGV) by its lesser packing density and small cell bodies. The medial division (MGM) consisting of large cells is evident medial to MGV and a clearly circumscribed suprageniculate nucleus (NS) is present. Most cells in NS are large, second only to those in MGM. Between MGM, NS, and DC lies a distinct group of small cells. This is the deep dorsal nucleus (Dd) (see Winer and Morest, 1983). Three regions can be distinguished within MGV, the densely packed and well ordered cells of $\mathrm{LV}$, the disordered arrangement of cells in OV and the flattened cells of VL. The small cells of BIN clearly distinguish this nucleus from MGM. There is little change in this arrangement rostrally ( $A 6.0$ in Fig. 1), except that the area occupied by Dd is much larger and 

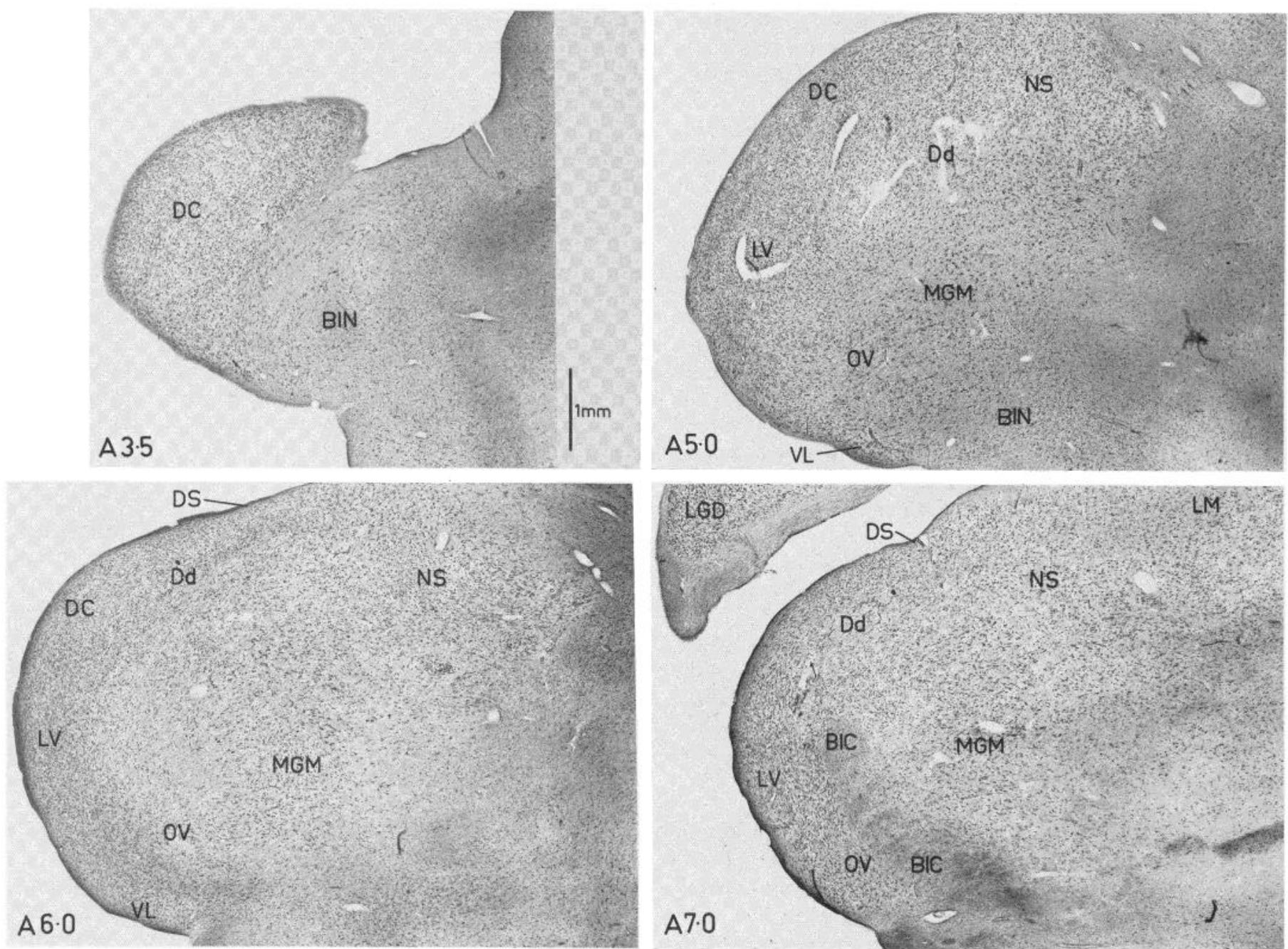

Figure 1. A series of four transverse sections through the MGB. These sections are tilted approximately $5^{\circ}$ toward dorsocaudal to ventrorostral from a true frontal plane, but this is of little consequence in viewing the MGB. The approximate anteroposterior planes of section are indicated. Sections were cut at $50 \mu \mathrm{m}$ and stained with thionine following fixation with glutaraldehyde $(2.5 \%)$ and paraformaldehyde vapors. $D S$, superficial dorsal nucleus; $L G D$, dorsal nucleus of the lateral geniculate body; $L M$, nucleus lateralis medalis.

DC is smaller. At this level the thin band of cells that form the superficial dorsal nucleus can be identified on the dorsal surface of the MGB.

The fibers of the brachium of the IC (BIC) are prominent and form a boundary between MGV and MGM in the anterior third of the MGB (A7.0 in Fig. 1); at this level the region dorsal to MGV is now occupied by Dd, which displaces DC anteriorly. Dd is easily distinguished from the highly ordered, and dense, pattern of cells in LV.

Thus, in this study, seven nuclei will be recognized as constituting the medial geniculate body: pars lateralis, pars ovoidea and ventrolateral nucleus, comprising the ventral division; caudodorsal, deep dorsal, and suprageniculate nuclei of the dorsal division; and the medial division. Additionally, the superficial dorsal nucleus and the nucleus of the brachium of the inferior colliculus can be distinguished. The former is considered to be part of nucleus lateralis posterior and the latter is a prethalamic nucleus (since it does not degenerate after removal of cortex; e.g., Rose and Woolsey, 1949).

\section{Results}

\section{Changes in physiological properties at anatomical borders}

When recording, "physiological" boundaries were found where the response properties of single units changed rapidly, with electrode depth, from one definable form to another. When electrode tracks were reconstructed histologically, the "physiological" boundary usually corresponded with the approximate position of a cytoarchitectural boundary. In some tracks the physiological transition took the form of an obvious and abrupt change in the strength of the auditory response and a dramatic alteration of unit properties, whereas in others a physiological boundary could be determined only after quantitative analysis of unit responses.

The electrode track summarized in Figure 2 exemplifies a situation where both quantitative and qualitative measures of the auditory response were used to distinguish physiological boundaries. The first part of the electrode track passed through a region with weak audi- 


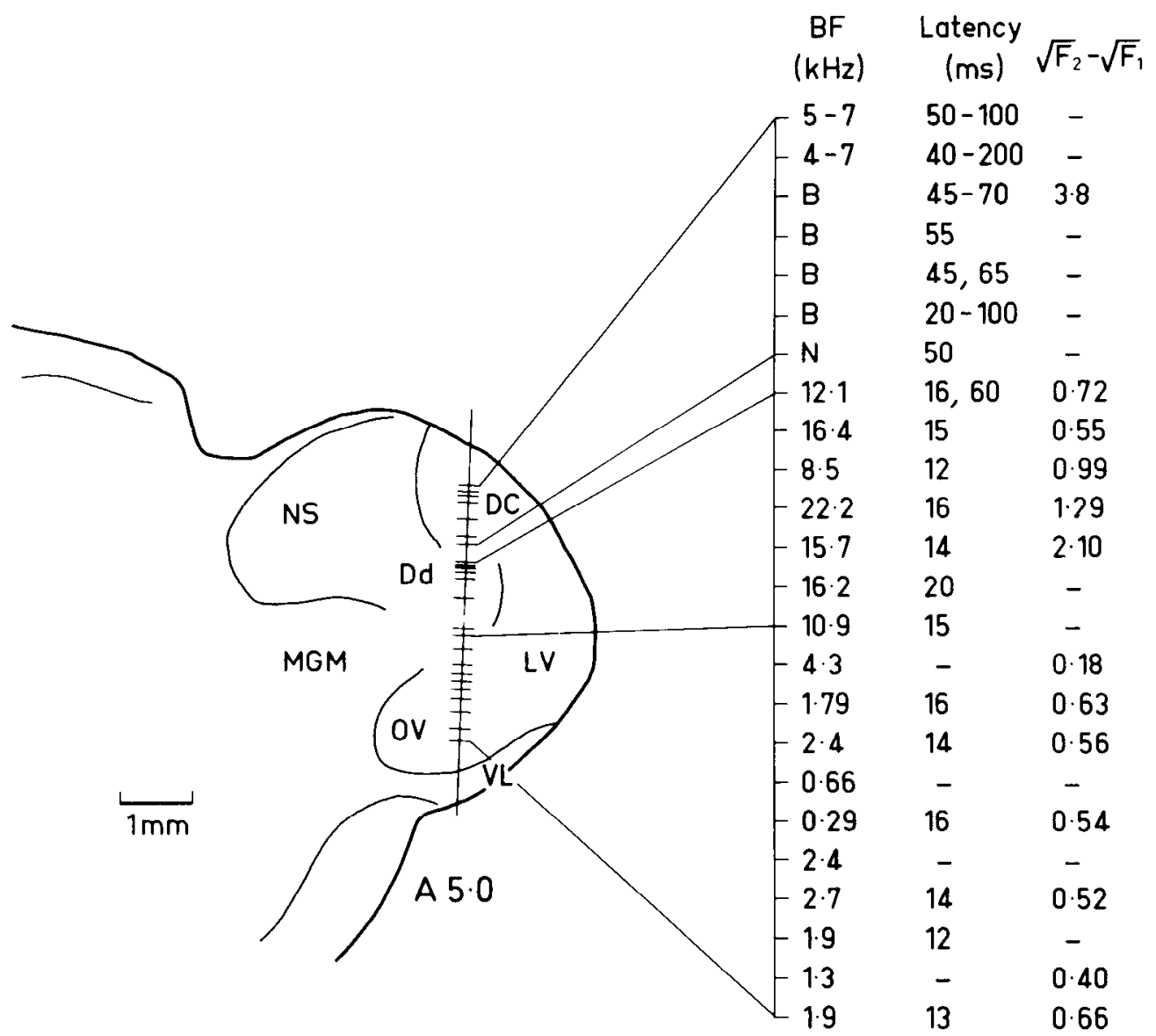

Figure 2. Outline drawing of a section (frontal plane A5.0) through the MGB of cat 8149 , indicating the position at which 24 single units were recorded. Unit properties of $\mathrm{BF}$, latency, and tuning $\left(\sqrt{\mathrm{F}_{2}}-\sqrt{\mathrm{F}_{1}}\right.$ in $\left.\mathrm{khz}^{1 / 2}\right)$ indicate two physiolngical transitions through which the track passes. These transitions correspond to boundaries between DC and Dd, and between Dd and OV.

tory drive containing units which gave long-latency responses, were broadly tuned, and habituated to repetitive stimulation. There is a clear transition from this region to the next where unit BFs could be readily determined and shorter-latency responses were more regular. Although BFs could be determined for units in this second region, the square root tuning measure (Calford and Webster, 1981) indicated that most of the units were not sharply tuned about their BF; all units were tuned to high frequencies and no tonotopic organization was apparent. A sudden change in $\mathrm{BF}$ from $10.9 \mathrm{kHz}$ to $4.3 \mathrm{kHz}$ between units 14 and 15 marked a transition to a region where units were more frequency selective, as indicated by $\sqrt{F_{2}}-\sqrt{F_{1}}$ values. In this final region the units responded consistently, to a narrow range of tone frequency at a short latency.

Reconstruction of the electrode track revealed that the first physiological boundary coincided with a transition from a small-celled region to a slightly larger-celled region, with approximately equal packing densities. The position of this boundary corresponds to the approximate position of the DC/Dd boundary. The second part of the track was medial to the densely packed cells of LV. The track continued into an area with a greater packing density at approximately the same position as the transition to the sharply tuned area. A circular arrangement of cells suggested this area to be $O V$.

Using a combination of physiological and cytoarchitectural criteria, an attempt was made to determine the subdivision in which each of 369 units was recorded, from 38 electrode tracks through the MGB. The sample is divided between seven nuclei, recognized as part of the $\mathrm{MGB}$, in the following manner: LV:86; OV:32; DC:97; Dd:32; MGM:45; NS:21; VL:12. A further 44 units could not be unequivocally assigned to a particular nucleus and are not used in the population analysis of auditory response properties.

\section{Discharge patterns}

An analysis of MGB discharge pattern types has been reported previously from this laboratory (Calford and Webster, 1981). In agreement with the previous study, the transient, single discharge pattern (onset response) was the dominant response type throughout the MGB. However, the types of discharge patterns varied considerably between subdivisions. The numbers of units in each subdivision displaying various discharge patterns 
are given in Table I; to enable comparisons between nuclei the data are presented in a proportional representation in Figure 3.

Some similarities between regions are immediately obvious. The samples from LV, OV, and Dd are dominated by very large proportions of transient, single responses, whereas only $50 \%$ of units in VL, DC, and NS have this type of pattern. A substantial proportion of VL, DC, and NS units (25 to $30 \%$ ) had transient responses which were not tightly stimulus locked, but varied in latency during the tone ("transient variable"). An appreciable number had multiple transient discharge periods during the tone, rather than only at its onset ("transient multiple").

Combined, these three transient discharge classes account for almost all of the sample of MGB unit discharge

TABLE I

Numbers of units displaying the indicated discharge pattern from each of the nuclei of the MGB

\begin{tabular}{lrrrrrrrr}
\hline & LV & OV & VL & DC & Dd & NS & MGM & Total \\
\hline Transient & & & & & & & & \\
$\quad$ Single & 68 & 22 & 6 & 40 & 25 & 10 & 21 & 192 \\
$\quad$ Multiple & 2 & 0 & 2 & 14 & 1 & 2 & 4 & 25 \\
Variable & 1 & 0 & 4 & 27 & 0 & 5 & 3 & 40 \\
$\quad$ Offset & 2 & 1 & 0 & 4 & 1 & 0 & 0 & 8 \\
Sustained & 11 & 5 & 0 & 1 & 1 & 0 & 15 & 33 \\
In.hibitory & 1 & 0 & 0 & 10 & 1 & 1 & 0 & 13 \\
Other & 1 & 0 & 0 & 1 & 1 & 0 & 0 & 3 \\
Total & 86 & 28 & 12 & 97 & 30 & 18 & 43 & 314 \\
\hline
\end{tabular}

patterns in this study (85\%). The samples form two groups, or clusters, one comprising LV, OV, and Dd and the other comprising VL, DC, and NS. Each group is dominated by transient responses, but those from the latter are commonly more complex.

Units recorded in MGM do not fit with either of these groupings. This sample includes a large proportion $(35 \%)$ of units which discharge in a sustained fashion, this being more than double the proportion of sustained units in any of the other MGB nuclei.

\section{Latency}

The minimum latency in this study was defined as the shortest latency of a unit response obtained when tested with tone bursts of increasing intensity, or white noise, if tones were ineffective, applied to the contralateral ear or binaurally. A rise/fall time of $4 \mathrm{msec}$ was routinely used for tone bursts.

The specification of minimum latency was not easy for those units which had nonmonotonic spike count versus intensity functions. These units reached a peak discharge level at a relatively low intensity, and thereafter increases in intensity reduced the response discharge level. This behavior contrasted with units having a monotonic spike count versus intensity relation, which either reached a plateau or displayed a response which continues to increase with intensity.

The distribution of minimum latencies for units from six MGB nuclei are presented in Figures 4 and 5. The shortest latencies were found in the samples of mono-

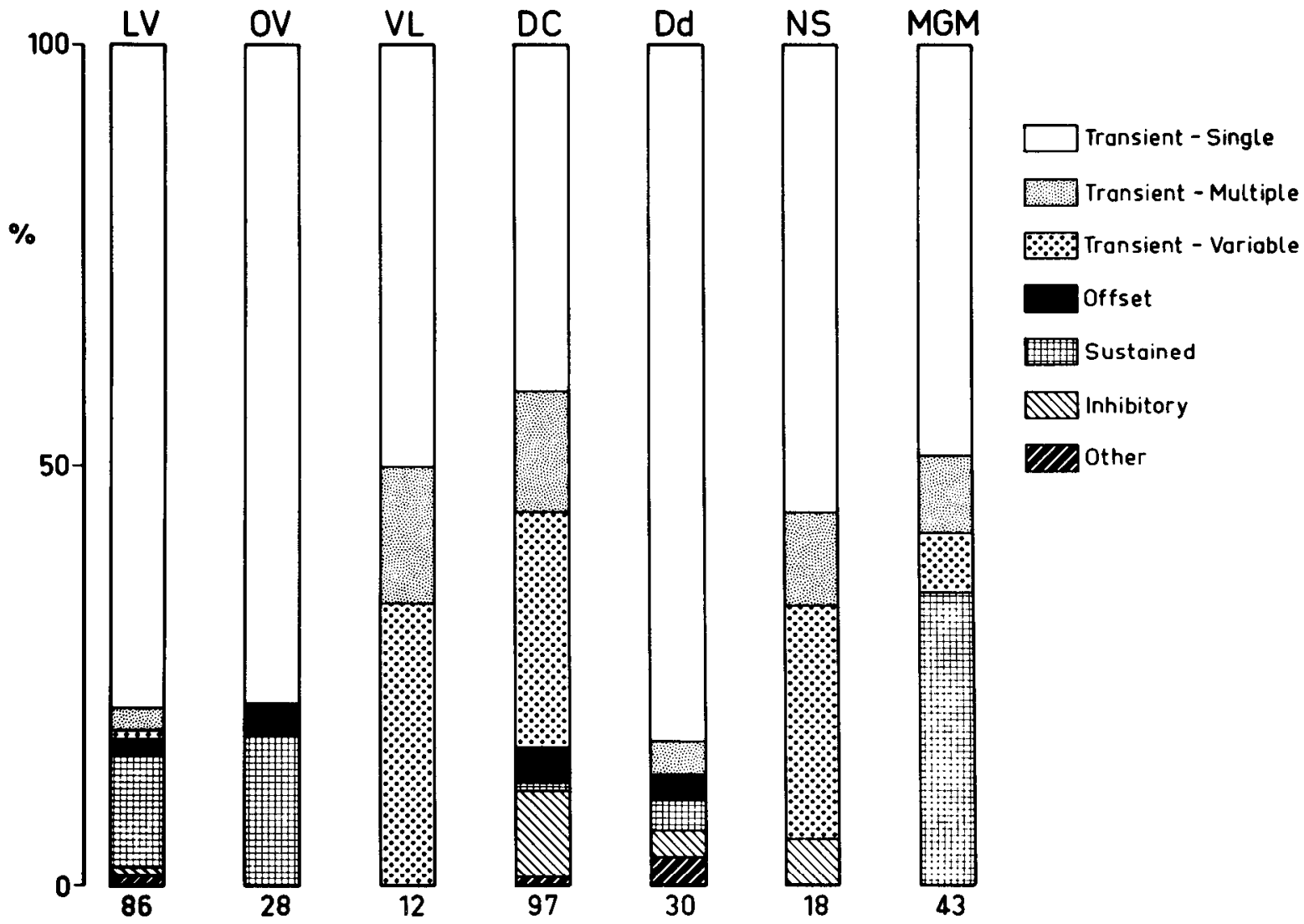

Figure 3. Proportional representation of the numbers of units displaying the indicated discharge patterns in the nuclei of the MGB. The numbers of units forming each sample are indicated below the respective bars. 

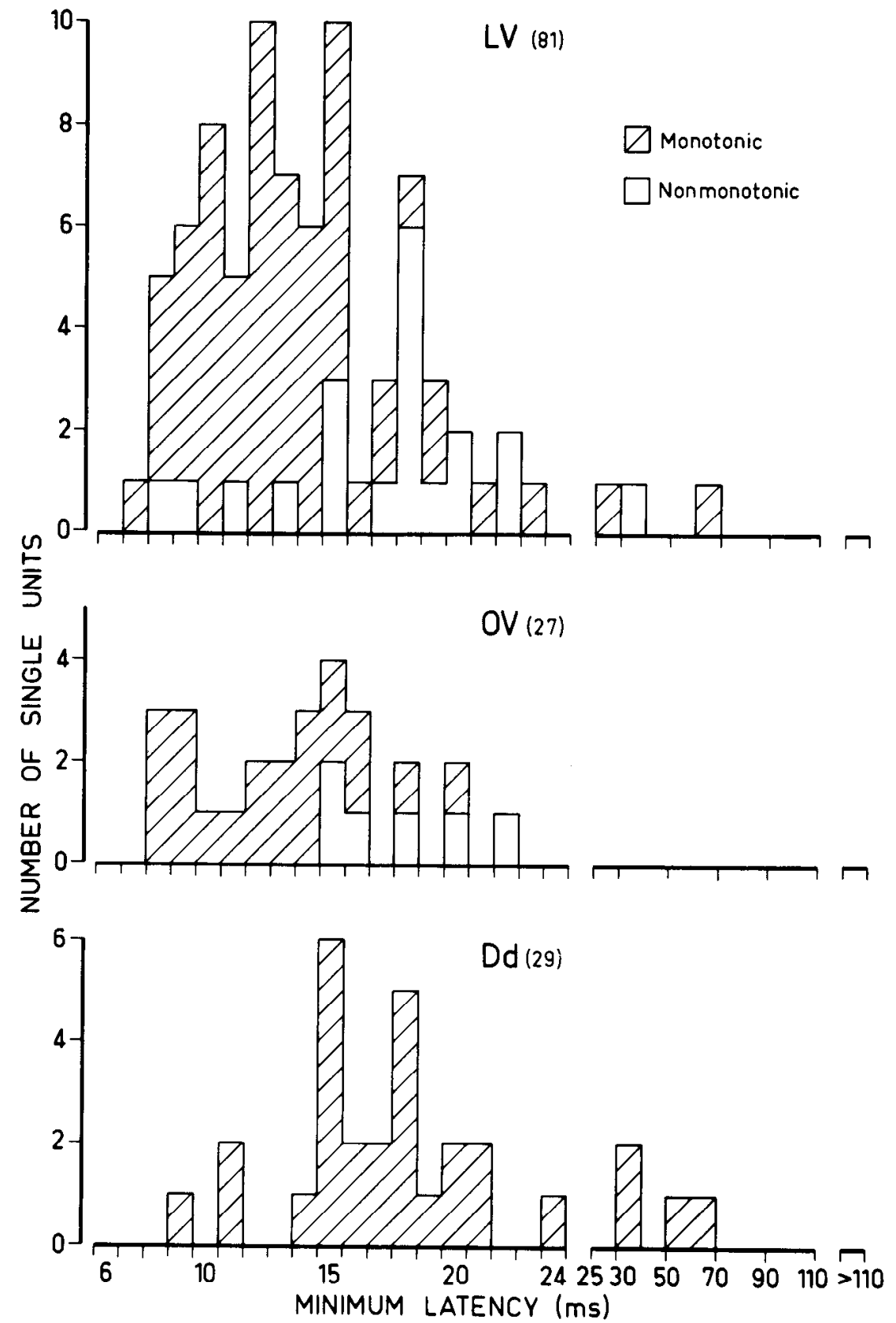

Figure 4. Histogram representation of the distribution of minimum discharge latencies to auditory stimulation for the indicated number of units in LV, OV, and Dd. The bin widths are staggered to provide a finer analysis at shorter latencies. The distributions of units with nonmonotonic spike count versus stimulus intensity relations are indicated separately.

tonic units from LV and OV; nonmonotonic units in these areas had slightly longer minimum latencies. Units in Dd had longer latencies than those in MGV, with a large proportion discharging between 15 and $20 \mathrm{msec}$. The samples from DC and NS were similar to each other, both having large numbers of very long latencies, whereas a broad spectrum of latencies was found in MGM. The sample from VL was small (12), but latencies were generally long and the distribution resembled that for NS.

The median test (Ferguson, 1971) was chosen as an appropriate nonparametric test for significance in these data, and a conservative $\alpha$ level of 0.01 was used to compensate for multiple testing effects upon the probability of a type I error. This test discriminated three pairs of samples: units in OV and LV had the shortest latencies, those in Dd and MGM were intermediate, and units in NS and DC had the longest latencies (Table II). Whereas the distributions of minimum latencies were similar for OV and LV and for NS and DC, the distributions for Dd and MGM were very different. This was indicated by the $F_{\max }$ test for homogeneity of variance (Winer, 1971) which showed that the MGM distribution had a significantly higher variance than that of Dd $\left(\mathrm{F}_{\max }\right.$ $=9 ; d f=38 ; p<0.01)$. 

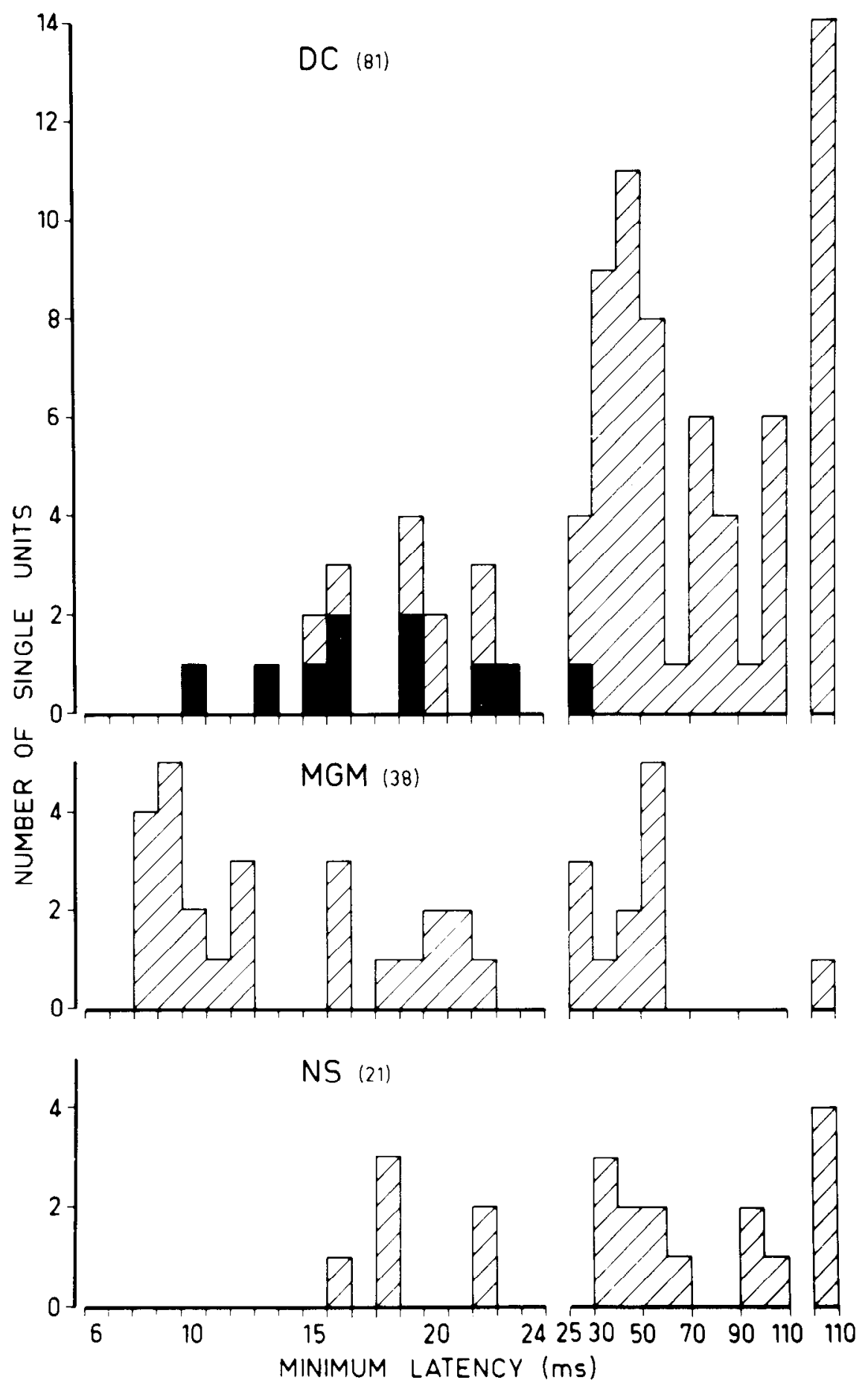

Figure 5. Histogram presentation of the distribution of minimum latencies for units from DC, MGM, and NS. The distribution for sharply tuned units in $\mathrm{DC}$ is indicated by the shading.

\section{TABLE II}

Medians of the distribution of minimum latencies for units from $M G B$ nuclei

Only monotonic units have been included from $\mathrm{LV}$ and OV samples (sample size, $n$ ).

\begin{tabular}{llllllc}
\hline & LV & OV & Dd & DC & NS & MGM \\
\hline Median (msec) & 12.6 & 12.5 & 17 & 51 & 47 & 18 \\
$n$ & 61 & 21 & 29 & 81 & 21 & 38 \\
\hline
\end{tabular}

\section{Frequency tuning}

The measure $\sqrt{F_{2}}-\sqrt{F_{1}}$, where $F_{2}$ and $F_{1}$ indicate the high and low limits of the tuning curve bandwidth $20 \mathrm{~dB}$ above the unit threshold, was used to give a quantitative assessment of the sharpness of frequency tuning for a large number of units. This measure was chosen because it has been shown that the square root transformation is independent of unit BF (Calford and Webster, 1981; 
TABLE III

Means $(X)$ and standard deviations (SD) of the $\sqrt{F_{2}}-\sqrt{F_{1}}$ scores $\left(k H z^{1 / 2}\right)$ for seven nuclei of the $M G B$

The total number of units $(n)$, the percentage too broadly tuned to allow scoring $(\% \mathrm{~B})$, and the percentage not responsive to pure tones $(\% \mathrm{~N})$ are also given.

\begin{tabular}{lllllllc}
\hline & LV & OV & Dd & DC & NS & VL & MGM \\
\hline $\mathrm{X}$ & 0.38 & 0.56 & 1.08 & 1.61 & 2.80 & 1.38 & 1.39 \\
SD & 0.18 & 0.19 & 0.41 & 1.10 & 0.52 & & 1.02 \\
$\% \mathrm{~B}$ & 0 & 0 & 5 & 69 & 68 & 64 & 41 \\
$\% \mathrm{~N}$ & 0 & 0 & 5 & 15 & 21 & 27 & 6 \\
$n$ & 39 & 24 & 21 & 78 & 19 & 11 & 32 \\
\hline
\end{tabular}

Calford et al., 1983; Whitfield, 1968; Whitfield and Purser, 1972). In addition, tuning discrimination was made for some units by briefly observing the discharge while altering the frequency of the stimulus to determine the minimum threshold and BF; a simple distinction was made between units sharply tuned about a single BF and other patterns (broadly tuned, limited dynamic range, etc.) classed as broad.

Table III presents a summary of the tuning characteristics of units in the samples from each nucleus. In addition to the $\sqrt{F_{2}}-\sqrt{F_{11}}$ scores, the percentages of units for which this measure is not applicable is also indicated. Thus $69 \%$ of DC units were classed as broad, but their tuning could not be quantified because they did not have a definable $\mathrm{BF}$ or they lacked a dynamic range greater than $20 \mathrm{~dB}$. Furthermore, $15 \%$ of DC units were unresponsive to tonal stimuli. Although it is difficult to compare the tuning properties from DC, NS, VL, and MGM, because the available $\sqrt{F_{2}}-\sqrt{F_{11}}$ scores may not be representative of frequency tuning in these areas, the values obtained from tuneable units indicate that these four regions contain units which are generally more broadly tuned than those of $\mathrm{LV}, \mathrm{OV}$, and Dd.

The distributions of $\sqrt{F_{2}}-\sqrt{F_{1}}$ scores for $L V, O V$, and Dd, from which adequate samples were obtained, are presented in Figure 6. It is clear that the distribution of scores in Dd is distinct from those of $I, V$ and OV, indicating that units in Dd are more broadly tuned. A small difference exists between the distributions of $\mathrm{LV}$ and $\mathrm{OV}$, although there is considerable overlap. As with the comparison of latencies, an analysis of variance cannot be used as a statistical test of significance for the $\sqrt{\mathrm{F}_{2}}-\sqrt{\mathrm{F}_{1}}$ distributions because the assumption of homogeneity of variance is invalidated $\left(\mathrm{F}_{\max }=5.2 ; d f=36\right)$. Consequently, multiple $t$ tests, using a $t$ test approximation (Welch test) for samples with unequal variances (Winer, 1971), a hierarchical order to avoid multiple testing, and a conservative $\alpha$ level of 0.01 were used to discriminate distributions. Both the LV/OV and OV/Dd $t$ tests showed significant differences between distributions $(t=7.3, d f=61, p<0.001$ and $t=5.11, d f=28, p$ $<0.001$, respectively).

The $\sqrt{F_{2}}-\sqrt{F_{1}}$ transformation was presented as a measure of frequency tuning, since it was found to be independent of unit BF. This property was also the case in the present samples for which correlation coefficients $(r)$ and linear regression slopes $(m)$ were calculated $(\mathrm{LV}$, $r=0.20, m=0.0019 \mathrm{kHz}^{-1 / 2} ; \mathrm{OV}, r=0.38, m=0.016$ $\left.\mathrm{kHz}^{-1 / 2} ; \mathrm{Dd}, r=0.058, m=0.005 \mathrm{kHz}^{-1 / 2}\right)$; all were found to be nonsignificant. Thus, it can be concluded that units in LV are more sharply tuned than those in OV and both groups of units are more sharply tuned than those in Dd.

A few units were found to be unresponsive to pure tonal stimuli but responsive to white noise or to transient stimuli. These units form significant proportions of the samples from DC, NS, and VL but are not present at all in $L V$ and $O V$, whereas a few of these units are seen in Dd and MGM (Table III). The proportions of these tonally unresponsive units further discriminate the highly frequency-selective units of LV and OV from the poorly frequency-selective responses in DC, NS, and VL.

\section{Relationship between latency and tuning}

The previous analyses suggest that for units in the MGB, certain response properties may be associated: e.g., long latency and broad tuning; short latency and sharp tuning. This relationship is illustrated in Figure 5 where the small proportion of sharply tuned units in DC $(12 \%)$ are seen to have shorter latencies (median $=17$ msec) than the broadly tuned units (median $=60 \mathrm{msec}$ ). This distinction between the tuning was made qualitatively for some units in DC and quantitatively, using the square root measure, for units from $\mathrm{LV}, \mathrm{OV}$, and Dd.

Figure 7 presents the relationship between minimum latency and the square root tuning measure for monotonic units from $\mathrm{LV}, \mathrm{OV}$, and Dd. The interaction of latency and frequency tuning clearly distinguishes the units in Dd from those in LV and OV. For any given latency most Dd units appear to be more broadly tuned than those in MGV, and the bulk of Dd units have longer latencies than units in MGV. Whereas units in OV display broader tuning than the bulk of the sample from $\mathrm{LV}$, there is no difference between these areas in terms of latency. A tendency exists for units with the shortest latencies also to have the sharpest tuning within a given subdivision, although this was only significant for $\mathrm{OV}$ when tested by a correlation analysis $(\mathrm{OV}, r=0.86, m=$ $0.038 \mathrm{kHz}^{1 / 2} \mathrm{msec}^{-1}, p<0.0001 ; \mathrm{LV}, r=0.28$, nonsignificant; Dd, $r=0.22$, nonsignificant). The relationship between latency and tuning is strong over the entire sample $(r=0.56, p<0.001)$, and the linear regression line is indicated on the figure $\left(m=0.055 \mathrm{kHz}^{1 / 2} \mathrm{msec}^{-1}\right)$. Thus, there is a clear monotonically increasing function relating unit minimum latency and unit frequency tuning $\left(\sqrt{F_{2}}-\sqrt{F_{1}}\right.$ scores), such that the shortest latency responses display the sharpest tuning.

\section{Binaural interaction}

Binaural interactions were classified in terms of the excitatory $(\mathrm{E})$, inhibitory $(\mathrm{I})$, or nil $(\mathrm{O})$ events evoked by stimulation of each ear, as in previous publications from this laboratory (Calford and Webster, 1981; Semple and Aitkin, 1979). Thus, for example, units classed as EI showed excitation to contralateral stimulation and inhibition of spontaneous activity with ipsilateral stimulation, whereas the $\mathrm{EO}(\mathrm{I})$ class was characterized by a nil effect of ipsilateral stimuli alone but an inhibition of contralateral excitation with simultaneous ipsilateral stimulation, and $\mathrm{EO}(\mathrm{F})$ indicates a facilitatory effect of binaural stimulation over and above contralateral alone. 
These categories can be collapsed into monaural input classes by considering the effect of each input seen in binaural interaction to reflect the mode of input. Hence EO(I) becomes EI and EO(F) becomes EE.

The numbers of units in the collapsed EE, EI, and EO monaural input classes for the areas with larger sample sizes are shown in a proportional representation in Figure 8. Each sample contains at least $60 \% \mathrm{EE}$ units and there is a general similarity between samples in terms of binaural interaction types.
Unit sensitivity to interaural phase delay was assessed by inverting the phase of the signal at one ear relative to the other, or by delaying the signal to one ear (without adding an onset-time delay). Delay-sensitive units were only found in $\mathrm{LV}$ and $\mathrm{OV}$, and all had BFs below $1 \mathrm{kHz}$. Fifty-five percent of $L V$ and OV units with BFs below 1 $\mathrm{kHz}$ were delay sensitive and all were classed as EE.

It would seem, from the present analysis, that the proportions of units displaying particular forms of binaural interaction provide little discriminatory infor-
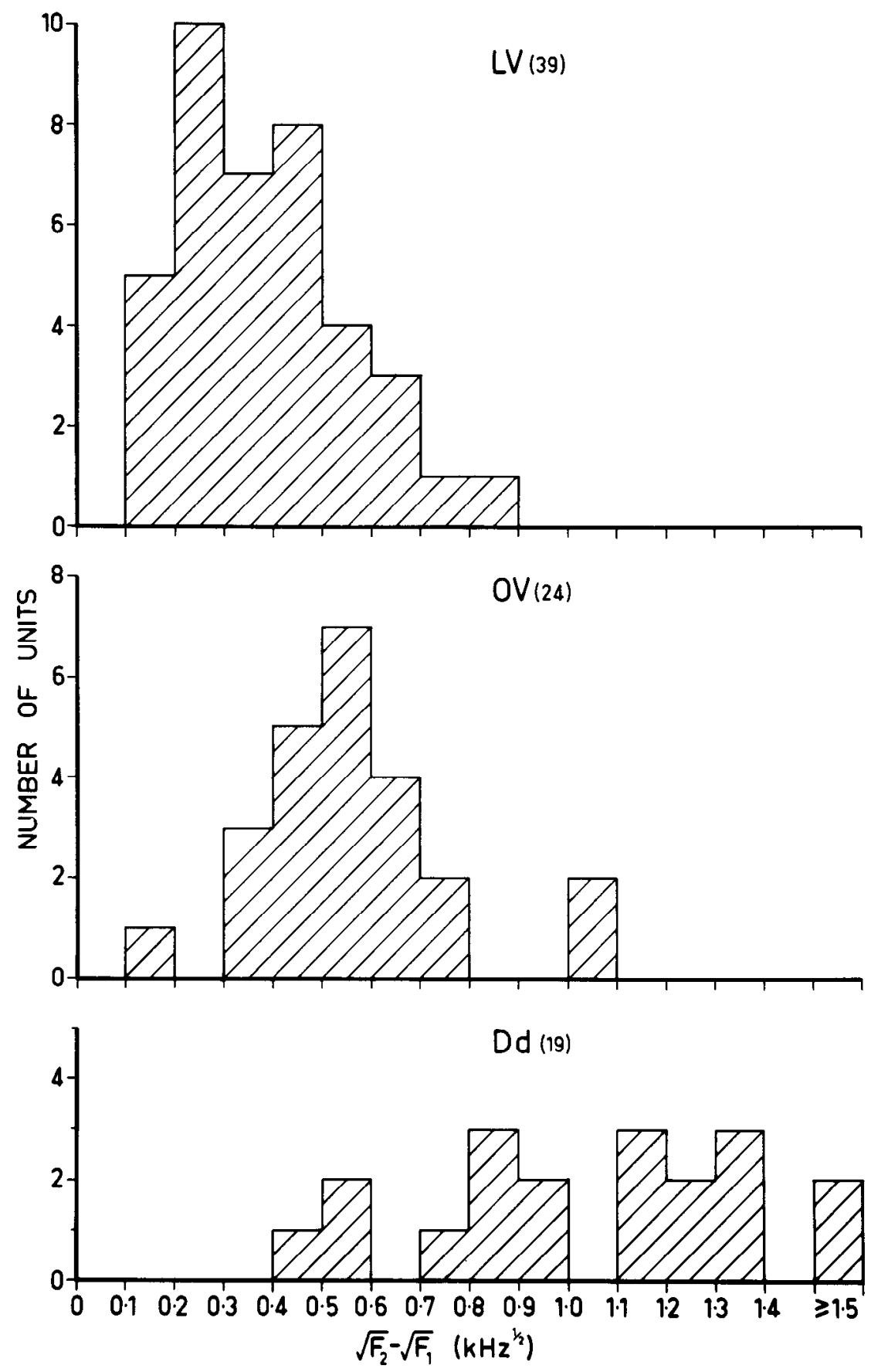

Figure 6. Distribution of $\sqrt{\mathrm{F}_{2}}-\sqrt{\mathrm{F}_{1}}$ scores from LV, OV, and Dd. Lower scores indicate sharper tuning properties. Each distribution is significantly different from the others. 


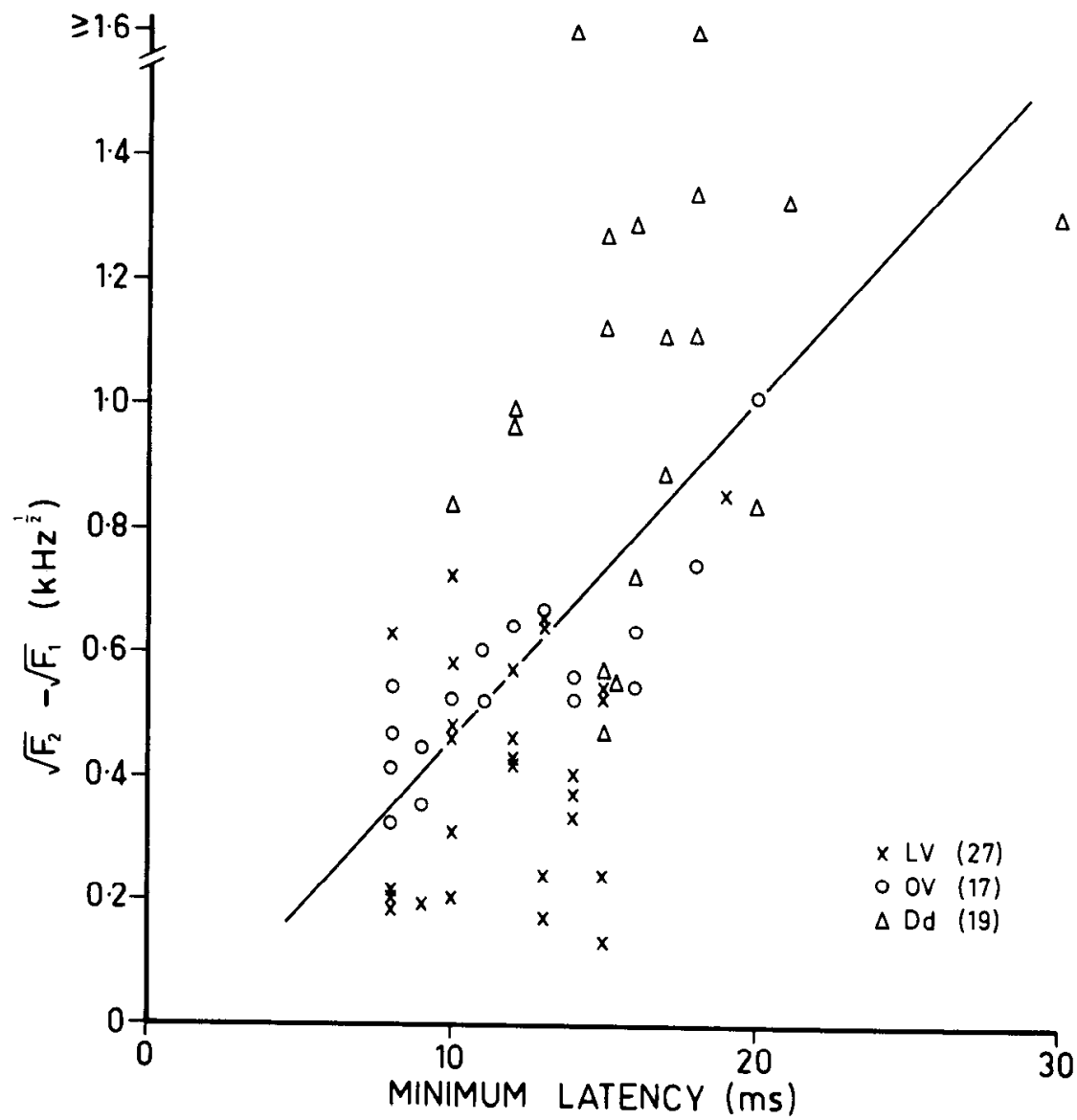

Figure 7. Scattergram showing the relationship of $\sqrt{\mathrm{F}_{2}}-\sqrt{\mathrm{F}_{1}}$ tuning scores to minimum latency for units from $\mathrm{LV}, \mathrm{OV}$, and $\mathrm{Dd}$. The solid line represents the least squares linear regression line for the entire sample. The numbers of units from each nucleus are indicated. Only units with monotonic spike count versus intensity functions have been used.

mation to aid in the physiological differentiation of subnuclei of the MGB.

\section{Tonotopic organization}

The tonotopic arrangement of LV has been well documented in previous publications (Aitkin and Webster, 1972; Calford and Webster, 1981). All electrode penetrations through this area displayed tonotopic sequences in accord with a dorsorostromedial to ventrocaudolateral/ high- to low-BF organization. OV has been recognized in this study by its ventromedial position in cytoarchitecturally defined MGV and by the absence of tonotopic sequences in tracks that pass through this area (Fig. 2).

The small proportions of units in NS, VL, and DC for which definite BFs were identifiable preclude the possibility of a tonotopic organization of these areas. Although most units in Dd have definable BFs, the sample contains only high BFs (all $>8.5 \mathrm{kHz}$ ). While it is possible that this represents a frequency bias in sampling, it would seem unlikely since five electrode tracks were made through $\mathrm{Dd}$, in separate animals, and a wide range of locations was covered. Furthermore, no tonotopic organization was found which would suggest the existence of a low frequency region of Dd. Only one track through
Dd showed any tendency toward a tonotopic sequence (Fig. 9), in that higher BFs are found for the units deeper in the penetration. This track passed through DC prior to Dd and terminated in the border area between MGM and LV (occupied mainly by BIC fibers) and gives no suggestion of a lower frequency Dd region.

BFs could be identified for over $50 \%$ of units in MGM, but no evidence of tonotopic organization was found.

\section{Habituation}

The consistency of responses to repetitive stimulation, or response strength, was assessed crudely by assigning units to one of four classes: (1) units were described as consistent if the discharge was maintained at a stimulus repetition rate of $1 \mathrm{~Hz}$; (2) units were described as rate sensitive if the discharge habituated with repetition of the same stimulus and remained so following minor changes in the stimulus parameters, but if the stimulus repetition was slowed sufficiently (usually $>0.2 \mathrm{~Hz}$ ), habituation did not occur; (3) units were described as novelty if habituation was not abolished by slowing the stimulus presentation rate (limited to $0.2 \mathrm{~Hz}$ ) but for which small changes in almost any stimulus parameter (laterality, intensity, frequency) restored discharge; (4) 


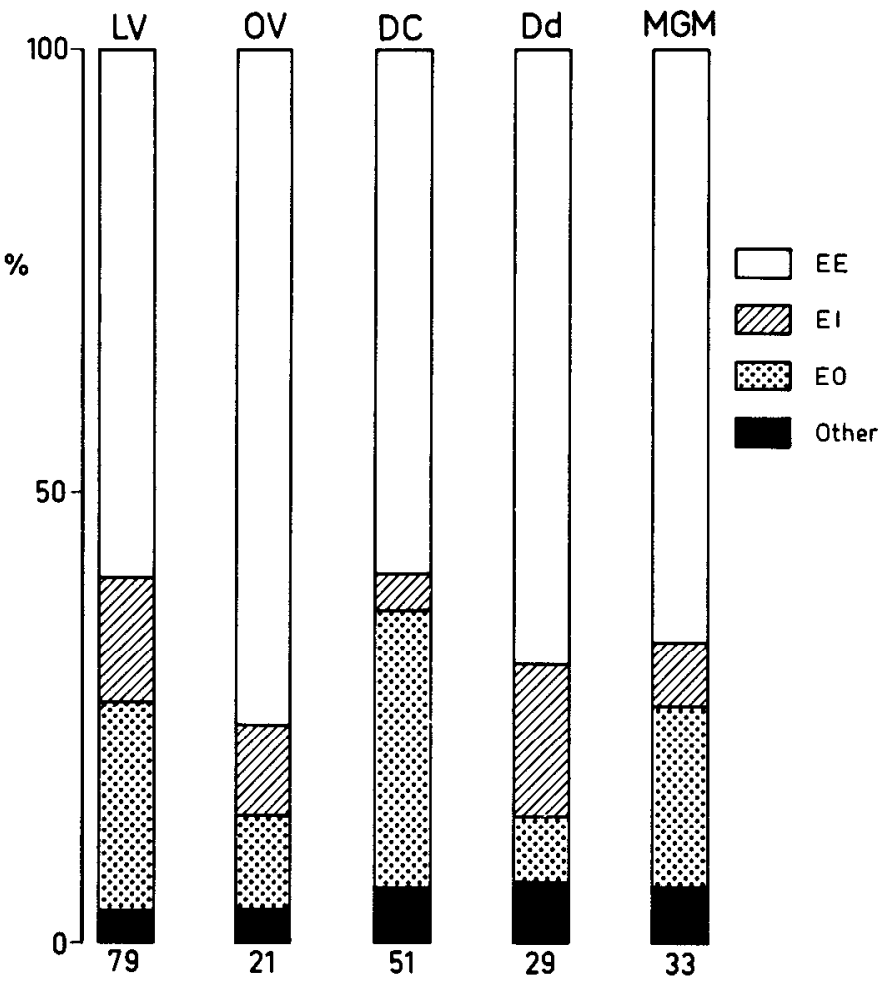

Figure 8. Proportional representation of the distribution of units, from five MGB subnuclei, among the three monaural input classes: $E E, E I$, and $E O$. All other combinations are grouped under Other. Contralateral responses are described first ( $E$, excitatory; $I$, inhibitory; $O$, no response) followed by ipsilateral.

units were described as labile if their response to acoustic stimulation was intermittent. All classes were found to be mutually exclusive. The proportions of units from each nucleus in each of the classes are shown in Figure 10. The ventral division nuclei, LV and OV, are clearly distinguished by their consistent responses. Other features are the high proportion of novelty units in DC and $\mathrm{VL}$, and variability of response types in NS and MGM.

\section{Summary of auditory response properties}

The ventral division ( $L V$ and $O V$ ). The most obvious features of MGV are its strict tonotopicity (in LV), the presence of strong background evoked activity ("swish"), and the homogeneity of auditory discharge characteristics over the entire sample. The vast majority of units respond regularly with a transient discharge at a short latency (onset responses) and are highly frequency specific. Units of MGV are as sharply tuned to tonal stimuli as any in the auditory pathway, with the most sharply tuned units located in LV and slightly broader tuning in $O V$. However, this was the only difference demonstrated between LV and OV, in terms of single unit responses to auditory stimuli.

The physiological boundary indicating the transition between $\mathrm{LV}$ and $\mathrm{OV}$ correlates with a change in BF. All electrode tracks through $\mathrm{LV}$ sampled tonotopic sequences of a form consistent with a mediodorsorostral to lateroventrocaudal/high- to low-frequency organization of unit BFS. In contrast electrodes passing through $\mathrm{OV}$, from any orientation, found rapid and marked changes in unit $\mathrm{BF}$ inconsistent with a simple tonotopic organization, although at any point all units appeared to respond to the same range of frequencies.

The caudodorsal nucleus (DC). The main feature of the DC sample is the high proportion of units with extremely long latencies and inconsistent responses to auditory stimuli. The majority of the units with purely inhibitory responses, from the total MGB sample, were located in DC. Most DC units respond with a transient discharge pattern with a high proportion showing multiple discharge periods or a variable latency, transient response. Only one-third of the units responded in a consistent fashion at a stimulus repetition rate of $1 \mathrm{~Hz}$.

A subgroup of DC units (12\%) display short latency, highly frequency-selective responses to tonal stimuli; properties similar to those found in MGV. The generally broad frequency responses in DC makes a tonotopic organization unlikely, but even when electrode tracks contained a substantial number of units with definable BFs no tonotopic sequences were evident in DC.

The deep dorsal nucleus ( $D d)$. The units in Dd have properties which are intermediate between those of the majority of units in DC and those of MGV. Thus, units respond at slightly longer latencies and are not as sharply tuned as those in LV and OV. Very few Dd units were unresponsive to tonal stimuli and most responded with a single, short latency, transient discharge. A distinctive feature of the Dd sample is that it contains only units with high BFs (all $>8.5 \mathrm{kHz}$ ).

The suprageniculate nucleus (NS). NS was found to contain units which responded at long latencies and showed poor frequency tu: ing. Their behavior is similar to that of the broadly tuned subgroup of DC, and $95 \%$ of NS units habituated to repetitive stimuli. Few NS units have definite BFs and a substantial proportion do not respond to tonal stimuli.

The medial division (MGM). Varied auditory responses were found in MGM. Most units were broadly tuned, but in terms of other properties MGM units covered the entire range of possible responses. Sustained discharge patterns were more common in MGM than in any of the other nuclei and were found in every penetration through the nucleus. Although auditory responses in MGM were strong and easy to define, the extreme variation between tracks does not allow the formulation of a set of response characteristics which differentiate the nucleus. In terms of the main aim of this investigation, it is fortunate that MGM is easily identified in Nissl-stained sections and that the surrounding nuclei have clearly different auditory responses to MGM.

The ventrolateral nucleus (VL). Only 12 units were sampled from VL, which is clear in Nissl-stained sections, but extremely small. All units displayed poor frequency selectivity and generally had very long response latencies. In all respects the units in the VL sample resemble those of the broadly tuned subgroup of DC units.

\section{Discussion}

This study has shown that subdivisions of the MGB defined by their differing cytoarchitecture and cellular 


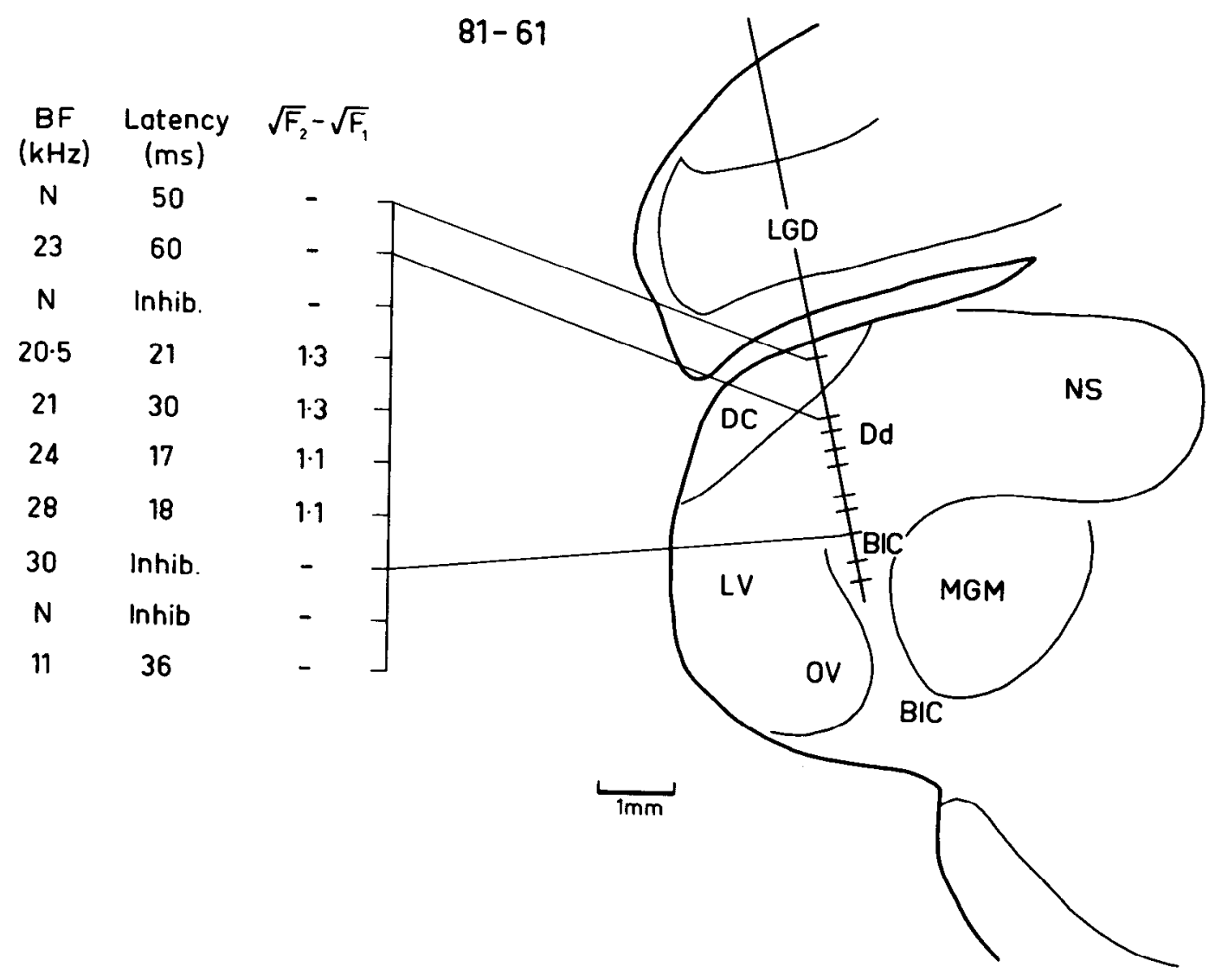

Figure 9. Passage of electrode track in experiment 81-61. Seven single units were isolated within Dd. The outlined section is of a near-frontal plane at approximately anterior $6 \mathrm{~mm}$. The $\mathrm{BF}$, minimum latency, and $\sqrt{\mathrm{F}_{2}}-\sqrt{\mathrm{F}_{1}}$ scores are given when available. $N$, responds to white noise but not to tonal stimuli; Inhib., stimulus suppresses unit discharges.

morphology (Morest, 1964, 1965a) contain units with differing auditory properties. A corollary to this finding is that, for the purpose of subsequent tracing studies of afferent input to the MGB, it is possible to make use of physiological criteria to aid in the placement of HRP injections in different MGB subdivisions.

Although the physiological data show many differences in auditory coding properties between subnuclei, discussion of the functional roles of these areas in the analysis of acoustic signals requires consideration of the afferent and efferent connectivity of MGB subdivisions. The determination of the ascending connections to the MGB is the subject of the following paper (Calford and Aitkin, 1983), and it is appropriate here to discuss the contribution of previous connectional studies to the differentiation of the MGB.

The ventral division. The distinction of the ventral division of the MGB on the basis of cellular morphology (Morest, 1964) is supported by almost every study examining connections of this region. The position, and the connections of the rostral two-thirds of the MGB from primary pathway nuclei (Moore and Goldberg, 1963; Powell and Hatton, 1969), and auditory fields (Butler et al., 1957; Diamond et al., 1958; Goldberg and Neff, 1961; Neff et al., 1956; Rose and Woolsey, 1949, 1958; Thompson, 1960; Woolsey, 1960) correspond well with later studies identifying MGV (Andersen et al., 1980a, b; Diamond et al., 1969; Kudo and Niimi, 1978,
1980; Niimi and Matsuoka, 1979; Niimi and Naito, 1974; Pontes et al., 1975; Raczkowski et al., 1976; Sousa-Pinto, 1973; Winer et al., 1977).

Most MGV principal cells have symmetrically tufted dendrites except for a small area at the ventral extreme (VL) (Morest, 1964). Recently, VL has been shown to have interconnections with the auditory cortex (Andersen, 1979; Andersen et al., 1980a) and midbrain (Andersen et al., 1980b) that are similar to those of DC. The connections between nonprimary cortical fields and DC/ VL differ markedly from the connections of $L V$ and OV with the tonotopically organized cortical fields (Andersen, 1979; Andersen et al., 1980a; Diamond et al., 1969; Jones and Powell, 1971; Sousa-Pinto, 1973; Winer et al., 1977) and midbrain nuclei (Andersen et al., 1980b; Kudo and Niimi, 1978, 1980).

The segregation of LV and OV (Morest, 1964, 1965a) is based upon the organization of the laminae formed by the parallel orientation of cells and the incoming tectothalamic and corticothalamic fibers. Laminae in LV parallel the external lateral and caudal surfaces of the MGB. The arrangement in OV is more complex, there being a roughly circular, but nonconcentric, organization of laminae. The topographic pattern of projection to or from LV shown in many studies is consistent with its laminar organization (Aitkin, 1976; Andersen, 1979; Andersen et al., 1980a, b; Kudo and Niimi, 1980; Merzenich and Colwell, 1975; Niimi and Matsuoka, 1979; Niimi and 

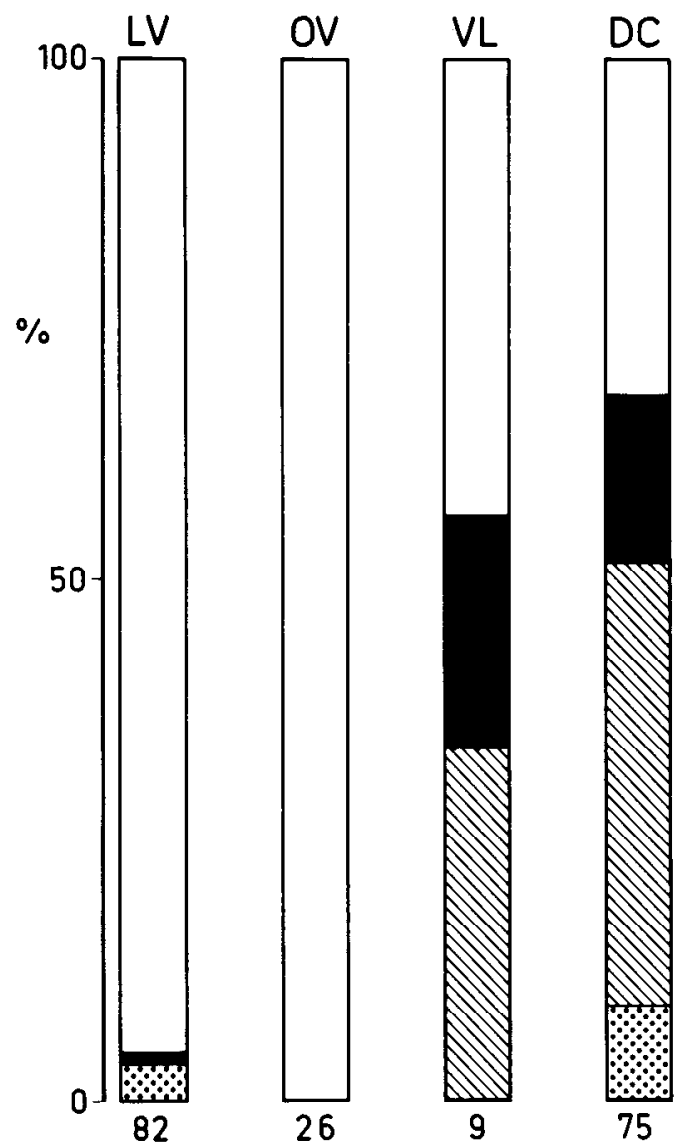

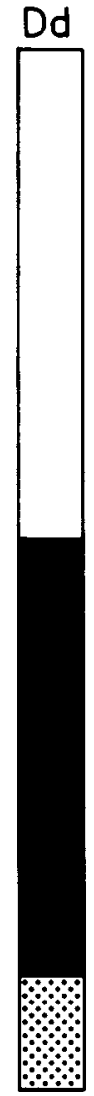

17

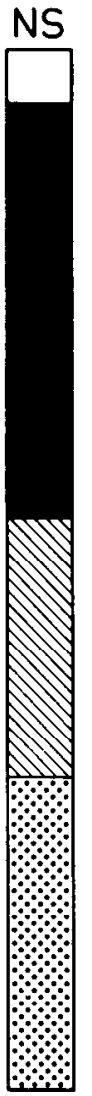

20
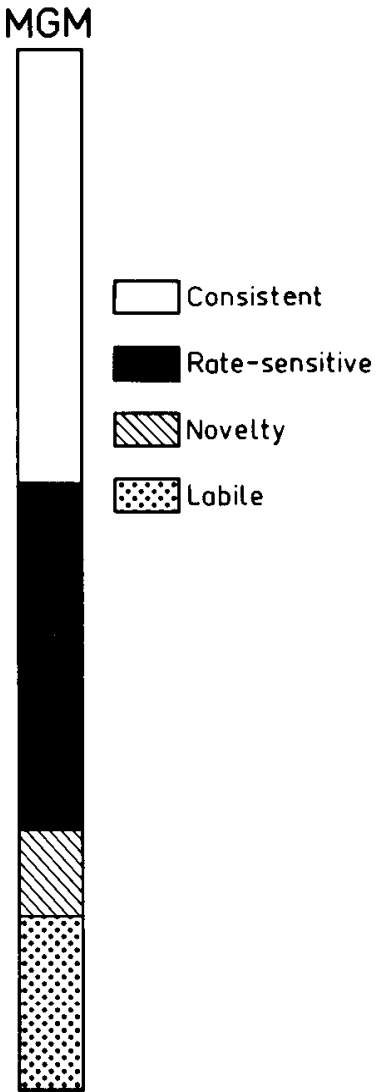

36

Figure 10. Proportional representation of the distribution of units among the four strength-of-response categories for the samples from the seven subnuclei of the MGB. The numbers of units in each sample are shown at the base of each bar.

Naito, 1974; Sousa-Pinto, 1973; Winer et al., 1977). Andersen and colleagues have shown a characteristic pattern in the projection to MGV from limited frequency regions of primary auditory cortex (AI) and the central nucleus of the IC (ICC), in which both LV and OV components can be distinguished (Andersen, 1979; Andersen et al., 1980a, b; Merzenich, 1981).

The dorsal division. Whereas early studies judged the principal division of the MGB to extend throughout the dorsal and ventral extents of the MGB (Berman, 1968; Moore and Goldberg, 1963; Rioch, 1929; Rose, 1942; Rose and Woolsey, 1949), there is now abundant evidence that a separate dorsal division can be distinguished (Andersen, 1979; Andersen et al., 1980a, b; Graybiel and Berson, 1980; Morest, 1964, 1965a, b; Niimi and Matsuoka, 1979; Pontes et al., 1975; Sousa-Pinto, 1973; Winer et al., 1977).

Morest's dorsal nucleus, or the caudodorsal nucleus (after Andersen, 1979), occupies the entire caudal tip of the MGB and extends dorsally over the caudal half of the structure. This nucleus most probably corresponds with the posterior third of the principal division defined, in early degeneration studies, by its connections with cortex (Butler et al., 1957; Diamond et al., 1958; Rose and Woolsey, 1949, 1958) and tectum (Moore and Goldberg, 1963; Powell and Hatton, 1969). Recently, Andersen and colleagues (Andersen, 1979; Andersen et al., $1980 \mathrm{a}, \mathrm{b}$ ) have shown that DC can be distinguished by its connections with the nontonotopically organized cor- tical field AII and by its input from the pericentral nucleus of the inferior colliculus. The deep dorsal nucleus sits ventral and anterior to DC and, although only a few studies have identified this area, there is supporting evidence that Dd has cortical and tectal connections which differ from those of DC (Andersen, 1979; Andersen et al., 1980a, b; Pontes et al., 1975; Winer et al., 1977).

Morest includes within his dorsal division the suprageniculate nucleus (NS), which occupies the region medial to Dd and DC and dorsal to MGM. A large number of studies have identified this region (Graham, 1977; Graybiel, 1972, 1973; Graybiel and Berson, 1980; Heath and Jones, 1971; Jones and Powell, 1971; Moore and Goldberg, 1963; Sousa-Pinto, 1973; Phillips and Irvine, 1979; Powell and Hatton, 1969; Rioch, 1929; Winer et al., 1977), although many different terms have been used to describe the area and definitions are not all equivalent. Many parcellations include the region occupied by NS within the posterior nuclear group (see Graybiel, 1973). NS can be distinguished from other nuclei of the MGB by its connections with fields in insular-temporal cortex (Jones and Powell, 1971; Sousa-Pinto, 1973) and by its sources of ascending afferents which include the deep layers of the superior colliculus (Berson and Graybiel, 1978; Graham, 1977).

The medial division. Most studies of the MGB identify a medial or magnocellular division. Recent studies have equated the magnocellular division (MGm), seen in Nissl stains, with Morest's (1964) medial division (MGM). The 
extent of these divisions varied between studies, and it has been suggested (Andersen, 1979) that parts of Dd were included in $\mathrm{MGm}$, as usually defined (e.g., Aitkin, 1973). Morest (1964, 1971) suggests that MGM defined by cellular morphology is larger than the earlier subdivision. However, it is difficult to accept MGM, as defined by Morest, as a single nucleus. Morest (1965b, 1971) describes projections from the caudal extent of MGM to the dorsal division of the MGB. It seems likely that this region is a separate nucleus and can be equated with BIN. This nucleus is well differentiated in Nissl-stained sections (Fig. 1; see also Berman, 1968). Most current workers identify BIN as a group of cells medial, ventral, and caudal to the MGB and within the BIC (Moore and Goldberg, 1963; Pontes et al., 1975; Powell and Hatton, 1969; Sousa-Pinto, 1973); others do not separate this region from the BIC (Kudo and Niimi, 1978), van Noort (1969) distinguishes rostral and caudal subdivisions of BIN.

Rose and Woolsey (1949) reported retrograde degeneration in MGm only after extensive lesions of auditory cortex, involving two or more fields. Later studies report MGM to project to cortical fields that ring the primary auditory areas (Heath and Jones, 1971; Graybiel, 1973) and to receive descending input from throughout auditory cortex (Diamond et al., 1969; Jones and Powell, 1971). These projection patterns distinguish MGM from the other nuclei of the MGB as does the finding that MGM receives ascending afferents from the external nucleus of the inferior colliculus (Kudo and Niimi, 1980).

\section{Conclusion}

There is close correspondence between boundaries, within the MGB, which are described by a change in the unitary auditory response and those described by cellular morphology (Morest, 1964). In addition, the division of the MGB into subnuclei defined jointly by these methods appears sufficient to account for all of the subdivisions suggested by the differential connections of subregions found in a wide variety of anatomical studies. Although it is difficult to describe the subnuclei of the MGB cytoarchitecturally, in normal Nissl-stained material, this study has demonstrated that a combination of electrophysiological recording and Nissl staining provides a basis for differentiating seven subnuclei.

\section{References}

Aitkin, L. M. (1973) Medial geniculate body of the cat: Responses to tonal stimuli of neurons in medial division. J. Neurophysiol. 36: 275-283.

Aitkin, L. M. (1976) Tonotopic organization at higher levels of the auditory pathway. Int. Rev. Physiol. 10: 249-279.

Aitkin, L. M., and W. R. Webster (1972) Medial geniculate body of the cat: Organization and responses to tonal stimuli of neurons in ventral division. J. Neurophysiol. 35: 365-380.

Aitkin, L. M., M. B. Calford, C. E. Kenyon, and W. R. Webster (1981) Some facets of the organization of the principal division of the cat medial geniculate body. In Neuronal Mechanisms of Hearing, J. Syka and I. M. Aitkin, eds., pp. 163181, Plenum Press, New York.

Andersen, R. A. (1979) Patterns of connectivity of the auditory forebrain of the cat. Ph.D. thesis, University of California, San Francisco.
Andersen, R. A., P. L. Knight, and M. M. Merzenich (1980a) The thalamocortical and corticothalamic connections of AI, AII and the anterior auditory field (AAF) in the cat: Evidence for two largely segregated systems of connections. J. Comp. Neurol. 194: 633-648.

Andersen, R. A., G. L. Roth, L. M. Aitkin, and M. M. Merzenich (1980b) The efferent projections of the central nucleus and the pericentral nucleus of the inferior colliculus in the cat. J. Comp. Neurol. 194: 649-662.

Andersen, R. A., R. L. Snyder, and M. M. Merzenich (1980c) The topographic organization of corticocollicular projections from physiologically defined loci in AI, AII and anterior auditory fields of the cat. J. Comp. Neurol. 191: 479-494.

Berman, A. L. (1968) The Brain Stem of the Cat. A Cytoarchitectonic Atlas with Stereotaxic Coordinates, University of Wisconsin Press, Madison.

Berson, D. M., and A. M. Graybiel (1978) Parallel thalamic zones in LP-pulvinar complex of cat identified by their afferent and efferent connections. Brain Res. 147: 139-149.

Butler, R. A., I. T. Diamond, and W. D. Neff (1957) Role of auditory cortex in discrimination of changes of frequency. $\mathrm{J}$. Neurophysiol. 20: 108-120.

Calford, M. B., and L. M. Aitkin (1983) Ascending projections to the medial geniculate body of the cat: Evidence for multiple, parallel auditory pathways through thalamus. J. Neurosci. 3: 2365-2380.

Calford, M. B., and W. R. Webster (1981) Auditory representation within principal division of cat medial geniculate body: An electrophysiological study. J. Neurophysiol. 45: 10131028.

Calford, M. B., L. M. Aitkin, C. E. Kenyon, and W. R. Webster (1980) Sources of ascending input and organization of single unit properties of the medial geniculate body in the cat. Proc. Aust. Physiol. Pharmacol. Soc. 11: 199P.

Calford, M. B., W. R. Webster, and M. N. Semple (1983) Measurement of frequency selectivity of single neurons in the central auditory pathway. Hear. Res., in press.

Diamond, I. T., K. L. Chow, and W. D. Neff (1958) Degeneration of caudal medial geniculate body following cortical lesions ventral to auditory area II in the cat. J. Comp. Neurol. 109: 349-362.

Diamond, I. T., E. G. Jones, and T. P. S. Powell (1968) The association connections of the auditory cortex of the cat. Brain Res. 11: 560579.

Diamond, I. T., E. G. Jones, and T. P. S. Powell (1969) The projection of the auditory cortex upon the diencephalon and brain stem of the cat. Brain Res. 15: 305-340.

Ferguson, G. A. (1971) Statistical Analysis in Psychology and Education, Ed. 3, McGraw-Hill Book Co., New York.

Goldberg, J. M., and W. D. Neff (1961) Frequency discrimination after bilateral ablation of cortical auditory areas. J. Neurophysiol. 24: 119-128.

Graham, J. (1977) An autoradiographic study of the efferent connections of the superior colliculus in the cat. J. Comp. Neurol. 173: 629-654.

Graybiel, A. M. (1972) Some fiber pathways related to the posterior thalamic region in the cat. Brain Behav. Evol. 6: 363-393.

Graybiel, A. M. (1973) The thalamo-cortical projection of the so-called posterior nuclear group: A study with anterograde degeneration methods in the cat. Brain Res. 49: 229-244.

Graybiel, A. M., and D. M. Berson (1980) Histochemical identification and afferent connections of subdivisions in the lateralis posterior-pulvinar complex and related thalamic nuclei in the cat. Neuroscience 5: 1175-1238.

Heath, C. J., and E. G. Jones (1971) An experimental study of ascending connections from the posterior group of thalamic nuclei in the cat. J. Comp. Neurol. 141: 397-426. 
Jones, E. G., and T. P. S. Powell (1971) An analysis of the posterior group of thalamic nuclei on the basis of its afferent connections. J. Comp. Neurol. 143: 185-216.

Kudo, M., and K. Niimi (1978) Ascending projections of the inferior colliculus onto the medial geniculate body in the cat studied by anterograde and retrograde tracing techniques. Brain Res. 155: 113-117.

Kudo, M., and K. Niimi (1980) Ascending projections of the inferior colliculus in the cat: An autoradiographic study. J. Comp. Neurol. 191: 545-556.

Merzenich, M. M. (1981) Some recent observations on the functional organization of the central auditory system. In Brain Mechanisms of Sensation, Y. Katsuki, R. Margion, and M. Sato, eds., pp. 3-19, John Wiley \& Sons., Inc., New York.

Merzenich, M. M., and S. A. Colwell (1975) Spatially ordered covergent projection from the auditory thalamus to and from AI in the cat. J. Acoust. Soc. Am. 57: 555.

Moore, R. Y., and J. M. Goldberg (1963) Ascending projections of the inferior colliculus in the cat. J. Comp. Neurol. 121 . 109-136.

Morest, D. K. (1964) The neuronal architecture of the medial geniculate body of the cat. J. Anat. 98: 611-630.

Morest, D. K. (1965a) The laminar structure of the medial geniculate body of the cat. J. Anat. 99: 143-160.

Morest, D. K. (1965b) The lateral tegmental system of the midbrain and the medial geniculate body: A study with Golgi and Nauta methods in cat. J. Anat. 99: 611-634.

Morest, D. K. (1971) Dendrodendritic synapses of cells that have axons: The fine structure of the Golgi type II cell in the medial geniculate body of the cat. Z. Anat. Entwicklungsgesch. 133: 216-246.

Neff, W. D., J. F. Fisher, I. T. Diamond, and M. Yela (1956) Role of auditory cortex in discrimination requiring localization of sound in space. J. Neurophysiol. 19: 500-512.

Niimi, K., and M. Matsuoka (1979) Thalamocortical organization of the auditory system in the cat studied by retrograde axonal transport of horseradish peroxidase. Adv. Anat. Embryol. Cell Biol. 57: 1-56.

Niimi, K., and F. Naito (1974) Cortical projections of the medial geniculate body in the cat. Exp. Brain Res. 19: 326-342.

Oliver, D. L., and W. C. Hall (1978a) The medial geniculate body of the tree shrew, 'I'upaia glis. 1. Cytoarchitecture and midbrain connections. J. Comp. Neurol. 182: 423-458.

Oliver, D. L., and W. C. Hall (1978b) The medial geniculate body of the tree shrew, Tupaia glis. II. Connections with the neocortex. J. Comp. Neurol. 182: 459-494.

Phillips, D. P., and D. R. F. Irvine (1979) Acoustic input to single neurons in pulvinar-posterior complex of cat thalamus. J. Neurophysiol. 42: 123-136.

Pontes, C., F. F. Reis, and A. Sousa-Pinto (1975) The auditory cortical projections onto the medial geniculate body in the cat. An experimental anatomical study with silver and autoradiographic methods. Brain Res. 91: 43-63.

Powell, E. W., and J. B. Hatton (1969) Projections of the inferior colliculus in cat. J. Comp. Neurol. 136: 183-192.

Raczkowski, D., I. T. Diamond, and J. A. Winer (1976) Organization of thalamocortical auditory system in the cat studied with horseradish peroxidase. Brain Res. 101: 345-354.

Ramòn y Cajal, S. (1955) Histologie du Système Nerveux de l'Homme et des Vertébrés, I. Azoulay, transl., Instituto Ramòn y Cajal, Madrid.

Rioch, D. McK. (1929) Studies on the diencephalon of carnivora. I. The nuclear configuration of the thalamus, epithalamus and hypothalamus of the dog and cat. J. Comp. Neurol. 49: 1-119.

Rose, J. E. (1942) The thalamus of the sheep: Cellular and fibrous structure and comparisons with pig, rabbit and cat. J. Comp. Neurol. 77: 469-524.

Rose, J. E., and C. N. Woolsey (1949) The relations of thalamic connections, cellular structure and evocable electrical activity in the auditory region of the cat. J. Comp. Neurol. 91: 441466.

Rose, J. E., and C. N. Woolsey (1958) Cortical connections and functional organization of the thalamic auditory system of the cat. In Biological and Biochemical Bases of Behavior, $\mathbf{H}$. F. Harlow and C. N. Woolsey, eds., pp. 127-150, University of Wisconsin Press, Madison.

Semple, M. N., and L. M. Aitkin (1979) Representation of sound frequency and laterality by units in central nucleus of cat inferior colliculus. J. Neurophysiol. 42: 1626-1639.

Sousa-Pinto, A. (1973) Cortical projections of the medial geniculate body in the cat. Adv. Anat. Embryol. Cell Biol. 48: 142.

Thompson, R. F. (1960) Function of auditory cortex of cat in frequency discrimination. J. Neurophysiol. 23: 321-334.

van Noort, J. (1969) The Structure and Connections of the Inferior Colliculus, van Gorcum \& Co., Netherlands Assen.

Whitfield, I. C. (1968) The functional organization of the auditory pathways. J. Sound Vib. 8: 108-117.

Whitfield, I. C., and D. Purser (1972) Microelectrode study of the medial geniculate body in unanaesthetized free-moving cats. Brain Behav. Evol. 6: 311-322.

Winer, B. J. (1971) Statistical Principles in Experimental Design, McGraw-Hill Book Co., New York.

Winer, J. A., I. T. Diamond, and D. Raczkowski (1977) Subdivisions of the auditory cortex of the cat: The retrograde transport of horseradish peroxidase to the medial geniculate body and posterior thalamic nuclei. J. Comp. Neurol. 176: $387-418$.

Winer, J. A., and D. K. Morest (1983) The neuronal architecture of the dorsal division of the medial geniculate body of the cat. A study with the rapid Golgi method. J. Comp. Neurol., in press.

Woolsey, C. N. (1960) Organization of the cortical auditory system: A review and synthesis. In Neural Mechanisms of the Auditory and Vestibular Systems, G. L. Rassmussen and W. F. Windle, eds., pp. 165-180, Charles C Thomas, Publisher, Springfield, IL. 